\title{
Brain Washing System-The System by Which the Wasting Molecule are Removed from Brain
}

Journal of Psychology and Neuroscience

Review Article

\author{
${\text { Mauro luisetto }{ }^{1 *}, \text { Muhammad Akram² }^{2} \text { and Areeba Imtiaz }}^{3}$ \\ ${ }^{I}$ IMA academy natural science branch Italy 29121 \\ ${ }^{2,3}$ Department of Eastern Medicine, Government College University Faisalabad, Pakistan \\ Submitted $\quad:$ November $22^{\text {nd }}, 2019$ \\ Accepted : December $4^{\text {th }}, 2019$ \\ Correspondence author \\ Published : December $10^{\text {th }}, 2019$ \\ Mauro Luisetto \\ Applied Pharmacologist \\ European Specialist Lab Medicine \\ Independent Researcher \\ Italy \\ Tel : +393402479620 \\ E-mail :maurolu65@gmail.com
}

\begin{abstract}
The central nervous system (CNS) is the only organ system of the body which lacks its own waste clearance or lymphatic system, a system which helps in removal of metabolic byproducts and waste solutes. Although the brain plays its role in $25 \%$ metabolism of body and comprises only $2 \%$ of the total body mass, this high metabolic load needs a proficient system for the removal of waste solutes and for maintaining homeostasis of brain environment. Well-depicted components of waste removalcomprise of perivascular fluid flow and phagocytic immune cell functions, nonetheless, the requirement for dynamic clearance of waste from the brain is getting progressively valued. Latest improvements in lymphatic vascular biologyconfrontthe recommendation that the braindeficitslymphatic removal system or anequivalent. In this review article, a recently discovered waste removal system, the glymphatic system and its functioning is discussed, keeping in view the experimental studies performed on rodents. The glymphatic system is peri-vascular network dependent of glial cells that serves as a pseudo-lymphatic system in the brain. In the pathway of glymphatic system, cerebrospinal fluid gains entry into brain by means of peri-arterial spaces, moves into the interstitial spaces through perivascular astrocytes and aquaporin-4 channels, and afterwards pushes the peri-venous waste of interstitial fluid (ISF) and its solutes into lymphatic vessels which eventually moves into systemic circulation. Thissystem plays significant role individually and also in combination with authentic lymphatic systemin drainage and getting clearance of wastes from the brain..
\end{abstract}

Keywords: Brain Washing System, Wasting Molecule, Neurodegenerative Disease, New Strategies, Toxicology

\section{Introduction}

The brain is a highly domineeringcontrollingorgan of the body, which takes input from all sensory organs of the body and plans the proper motoroutput accordingly, considering learning and memory, and taking into consideration running fixes to keep up dynamic capacity. This coordination is carried out by neurons and they use gradients for ions and flow of charges to create potential fundamental changes in the synaptic interconnections, including action potentials that transmit along axons, and synaptic potentials produced in post-synaptic membranes by transmitters released from the presynaptic neuron [1].

The neural microenvironment is separated from blood while permitting efficient exchange of vital gases, nutrients and metabolic waste products and proficient evacuation of bigger waste products and cell debris to maintain homeostasis of the
The neural microenvironment is separated from blood while permitting efficient exchange of vital gases, nutrients and metabolic waste products and proficient evacuation of bigger waste products and cell debris to maintain homeostasis of the brain and its surroundings. The lodging of the sensitive brain tissue inside the skull gives some mechanical protection from injury, however 'buffer zones' enabling the brain to glide in an appropriatefluid are additionally required. The modern mammalian brain accomplishes these by compartmentalization that permits dynamic exchange crosswise over key interfaces [6]. In the CNS, the blood-brain-barrier (BBB) prevents entrance of fluid and unregulated proteins, nevertheless, some waste products still succeed to gain entry into the interstitial spaces 
of brain due to the high metabolic rate [10,36]. The CNS is the only system of the body which lacks properly developed and efficient lymphatic system for the drainage and removal of waste products [33].

A recently discovered system identified as the"glymphatic system" is known to remove waste products and cell debris from brain. It derives its name from the 'glial cells' and the 'lymphatic system, this elucidates how the most sensitive and efficient organ of human body removes excessive fluids and waste products without any lymphatic system. This system covers the whole brain tissue and assists exchange of interstitial fluid (ISF) with cerebrospinal fluid (CSF) to clear interstitial wastes from brain parenchyma which is eventually cleared by cervical lymphatic vessels [15].

\section{The Brain System}

The human brain is very complexly protected by meninges; the outer coverings of brain which are three in number viz. dura mater; the outer most, arachnoid mater; the middle layer and the inner most; pia mater. Cerebrospinal fluid (CSF) which circulates inside these meninges is primarily a circulating plasma which is present unequally inside the ventricular sites (the two lateral, third and fourth ventricles) inside brain and inside extra ventricular spaces outside the brain and spinal cord (subarachnoid space) The extracellular space (ECS) between glial cells and neurons is filled by the interstitial fluid (ISF) $[23,30]$. The composition of interstitial fluid (ISF) is much similar to that of CSF $[27,29]$.

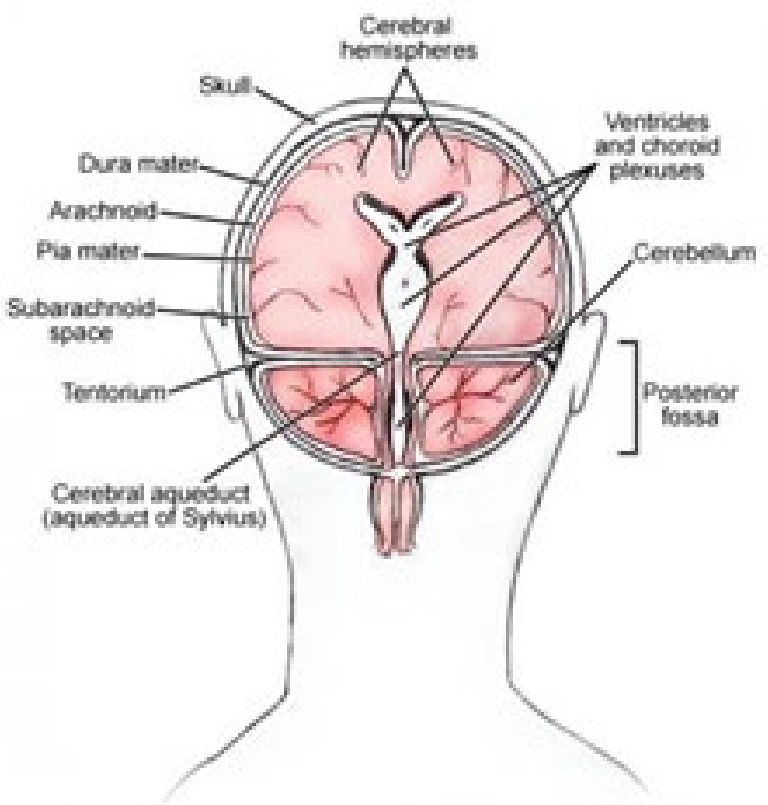

Fig. 1

\section{CSF-The Cerebrospinal Fluid}

A clear and transparent ultra-filtrate of plasma that is released up to $80 \%$ by the epithelial cells of choroid plexus and $20 \%$ by the other structures like brain parenchyma and ependyma, this fluid is the cerebrospinal fluid or CSF. It is secreted into the ventricle sites of brain $[5,10,13]$. As compared to blood,
CSF has low concentration of proteins and potassium $(\mathrm{K}+)$, on the other hand it has higher content of sodium $(\mathrm{Na}+)$, chloride (Cl-) and magnesium (Mg++). Water content of CSF is $99 \%$ as compared to $92 \%$ concentration of water in blood plasma [5]. The CSF after being produced by choroid plexus moves into ventricle sites and cisterns and then circulates in the whole brain tissue. It then moves into subarachnoid space and is ultimately absorbed through the subarachnoid villi into blood [8]. It acts as a sink for brain extracellular solutes and metabolic waste products which are not able to be removed by the passive diffuse across blood-brain-barrier [12,26].

The CSF has not only unidirectional flow but it also flows in pulsatile manner and in to and fro movement, this helps in local fluid exchange between blood, CSF and ISF. The other important factors which play important role in maintaining homeostasis of CSF and water content in brain include astrocytes, aquaporins (AQP) and other membrane transporters [4].

\section{Blood Brain Barrier-BBB}

Blood-brain-barrier or BBB is an exclusively permeable membrane or sheath whichsplits the circulating blood from the brain and ECF (extracellular fluid) in the CNS. Recent studies have revealed that the function of BBB as 'barrier' is due to highly regulated and complex molecular and cellular transport processes, this permits the exchange of water content, solutes, large molecules and even some cells across the membrane $[1,2,21]$. The BBB comprises of endothelial cells of blood capillaries that are coupled with tight connections, while the blood-CSF barrier forms amongst the epithelial cells of choroid plexus [5].

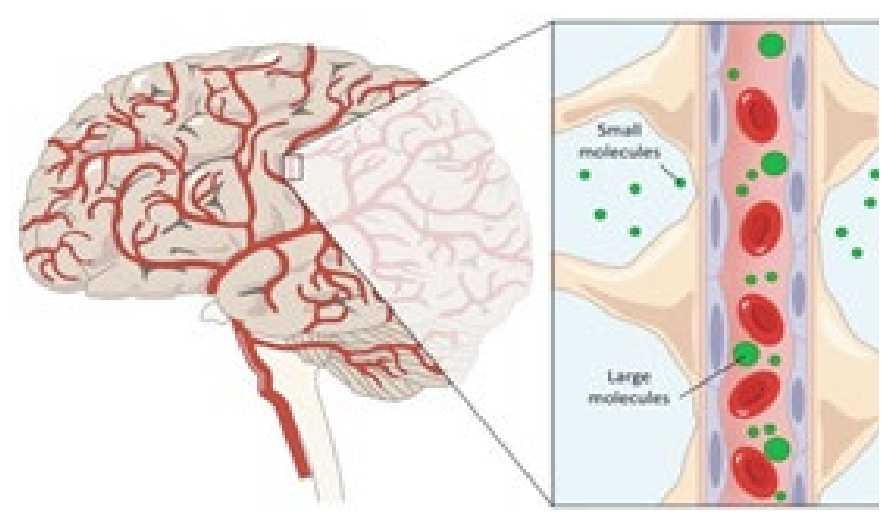

Fig. 2

The latest apprehension of BBB physiology was further enhanced by the discovery which tells that the function of $\mathrm{BBB}$ can be controlled and regulated by the cells that surround the capillaries. In respect to the contribution of pericytes, astrocytes, microglia and neurons, the blood-brain-barrier is better labelled as a 'neurovascular unit' [22]. When considering CSF physiology and its role in protection of brain tissue, the role of astrocytes is the most significant, as astrocyte end-feet cover the whole capillary surface, these leave small intercellular clefts behind [18]. Therefore, an extra additional barrier is formed by the astrocytes around the cerebral capillaries [28]. 


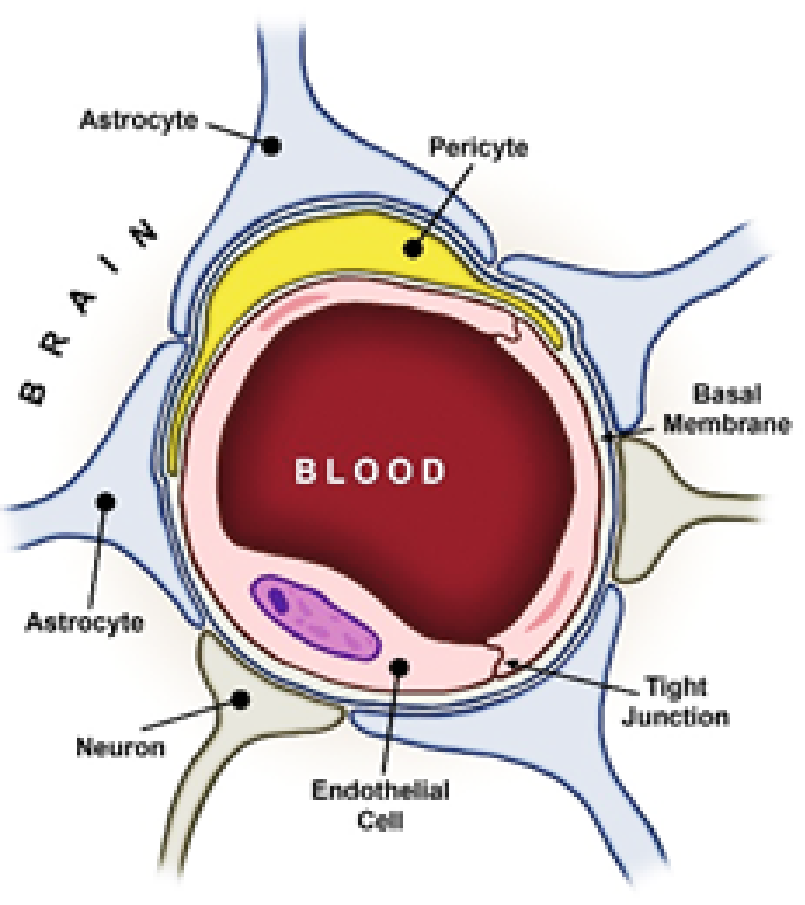

Fig. 3

\section{Brain Vasculature and Perivascular Space}

The vasculature of brain has a few special characteristics that differentiate it from the vasculature of the remainderof the body. The arterial cerebral vessel comprises of ananteriorand a posterior cerebral vessels provided by the interior carotid arteries and the vertebral arteries, correspondingly. The anterior vessels, which incorporate the anterior and middle cerebral arteries, connectto the posterior vessels, the basilar artery and posterior cerebral arteries, by means of anterior and posteriorcommunicating arteries at the Circle of Willis. From the Circle of Willis, the anterior vesselsperfuse the more young parts of the brainwhich include the neocortex of the cerebral hemispheres, whereas the posterior vessels perfuse the cerebellum and brainstem $[17,24]$.

At the cortical surface, cerebral arteries stretch out into pial arteries going through the subarachnoid space and the sub-pial space $[35,37]$. As pial arteries plunge downwards into the brainsystem, they change into infiltrating arterioles and make a perivascular space, termed as the VirchowRobin space [17]. The Virchow-Robin spaces are loaded up with CSF and surrounded by a leptomeningeal cell layer on the inner wall confronting the vessel and also on the outer wall confronting perivascular astrocytic end-feet. An interesting component of the CNS vasculature is that all vessels inside the brain parenchyma are encompassed by astrocytic vascular end-feet. These vascular end-feet make the outer wall of the perivascular space looking like a doughnut shaped passage encompassing the whole vasculature. As the piercingarterioles narrowdeep down in the brain parenchyma, the Virchow-Robin spaces become ceaseless with the basal lamina. The basal lamina is a thin sheet of loose extracellular matrixbasically composedof laminin, collagen type IV, fibronectin and heparin sulfate proteoglycan [7,31].

In addition to establishing a lessresistant pathway for CSF influx, the perivascular spaces are likewise significant destinations for conveyance of energy substrate and regulating blood stream. In diseasedstates, for example, stroke, the natural inflammatoryresponse starts and edema develops in the perivascular spaces. (Del Zoppo et al., 2015) Blood moves from the cerebral capillaries into the postcapillary venules where expanded basement membranes of endothelial cells and astrocytes give a bigger CSF-filled perivascular space [9].

\section{Glymphatic System-Clearance System of Brain}

Removal of waste from the CNS is indispensable for maintenance of brain homeostasis throughout the life. Two interconnected, powerful networkswhich are recently revealed, which may give new data regarding the problem of how the braincopes withwaste clearance without any authentic lymphatic system. The glymphatic network serves as the brain's 'front end'waste removingsystem that incorporates a perivascular network for transport of CSF $[11,12]$.

Glymphatic perfusion of the CNS fulfills numerous needs in neurophysiology. The glymphatic pathway is significant for the brain-wide supply of nutrients, explicitly glucose, the flow and distribution of apolipoprotein $\mathrm{E}$ isoforms secreted by the choroid plexus, and even astrocytic paracrine signaling with lipid molecules; in any case, its most major partis the 'lymphatic'function it serves in clearing extracellular metabolites and waste items from the parenchyma into the CSF $[3,11,13,32]$.

The glymphatic system and waste removal process is a 3 step sequential procedure as follows:

- CSF constantly circulates from the basal cisterns into the subarachnoid space encompassing the cerebral hemispheres, it then enters the peri-arterial spaces in a bulk flow pattern.

- CSF is impelled from the peri-arterial compartment into the interstitial fluid space acceleratedby aquaporin 4 (AQP4) water channels on astroglial end-feet which ease rapid exchange of water across the membranes, a procedure empowering mixing of CSF and ISF and removal of waste solute. 
- Themixture of CSF and ISF containing interstitial waste solutes is consequently moved towards the peri-venous compartment of the larger central veinsfrom where it ultimately moves into lymphatic vessels and systemic circulation [12].

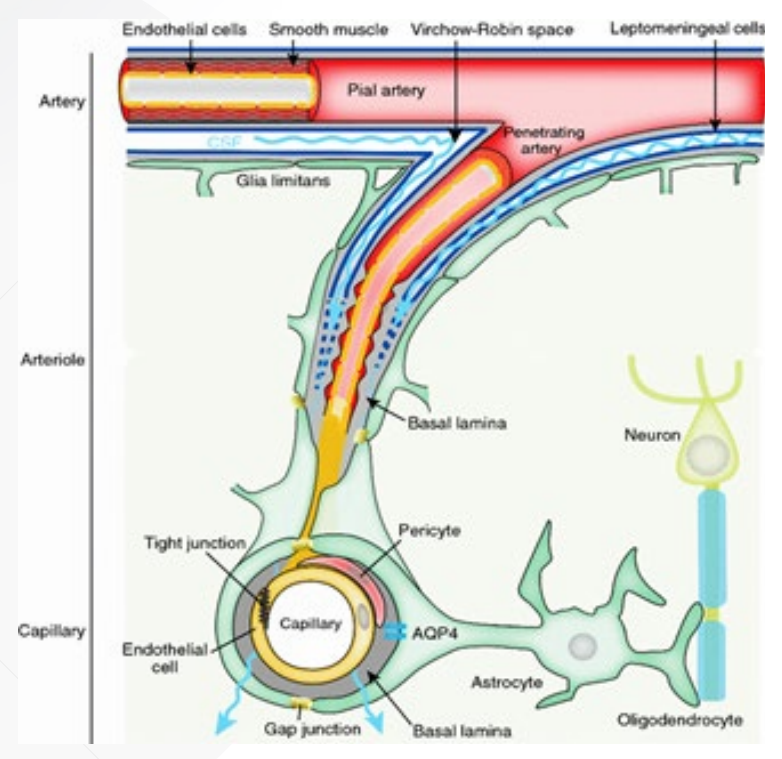

Fig. 4

The anatomical structure and physiology of the neurovascular unit permit two-directional transmissionamong the microvasculature and neurons, with astrocytes performing intermediate functions. Pial arteries in the subarachnoid space turn out to be infiltrating arteries after plunging into the brain parenchyma. The perivascular space around infiltrating arteries is the Virchow-Robin space. As the entering arteries divide into arterioles and capillaries, the VirchowRobin spaces start narrowing and lastly vanish. CSF from the Virchow-Robin spaces moves into the perivascular spaces around capillaries, arterioles and venules where the extracellular matrix of the basal lamina gives a progression of the fluid space. Astrocytic vascular end-feet expressing aquaporin 4 (AQP4) encompass the whole vasculature and structure the limit of the perivascular space [13].

Latest advancements have shown that exchange of ISF and CSF is continuous. Influx of CSF down peri-arterial spaces aids this exchange procedure [12]. Movement of CSF into the brain parenchyma moves ISF contained in the tissue towards the peri-venous space around the large deep veins $[14,19]$. This profoundly polarized macroscopic system of convective fluidmoves with quick exchange of CSF and ISF is titled as the glymphatic system due to resemblance to the function of lymphatic system in the peripheral tissues, and on the significant role of glial AQP4 channels in the fluid transport [12]. Thus the para-vascular glymphatic pathway directed by AQP4-dependentmassive flow establishes a significant interstitial fluid and solutes removal pathway in the brain parenchyma [32].

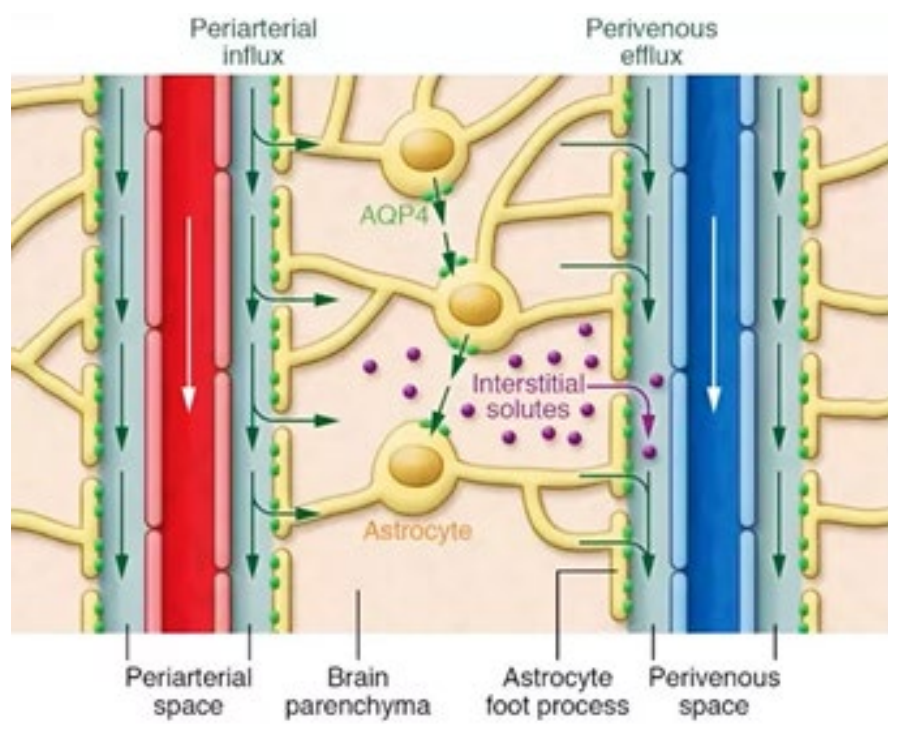

Fig. 5

Strangely, the glymphatic system works most proficiently during rest when there is a $60 \%$ expansion in interstitial space because of a decrease in the size of cells inside the brain and the effectiveness of this clearancemechanism is additionally diminished in the brain with increasing age, recommending possibly significant roles in age-related illnesses [16,34]. Recent studies show that sleep affects glymphatic system positively; i-e; sleep enhances clearing of solute wastes especially amyloid- $\beta$ from brain. This is most probably happened due to the increase in cortical ISF space up to $40-60 \%$ as compared to the cortical ISF space during wakefulness. Under normal wakefulness, the cortical ISF space is comparatively lesser to solute waste transport, on the other hand, it expands during sleep allowing more CSF to enter glymphatic pathway and continue exchange with ISF [34].

\section{In Vivo Study of Glymphatic System}

In a recent in vivo experiment, fluorescent dextran were injected into the cisternal CSF of rodents, and in a course of 30 minutes there was powerful perivascular marking $[12,25,26]$. With the utilization of intra-vital two-photon microscopy, the fluorescent CSF tracers quickly showed up, as quick as 5 minutesafter infusion, inside the perivascular spaces of surface arteries and afterward, over the resulting 25 minutes, moved gradually more deep into the parenchyma inside the perivascular spaces of infiltrating veins. Therefore, influx of CSF into perivascular spaces was approved, and 
furthermore a direction to this smooth flow of fluid was illustrated, with CSF flowing into the brain only within periarterial spaces and ISF leaving the brain within peri-venous channels [12].

Invivo two-photon MRIstudy of tiny fluorescent tracers endorsed explicit visualization of the speedy peri-arterial solute influx into the cortex and exhibited that higher molecular weightdyes were stuck on the gaps between astrocytic end-feet comprising a physical hindrance to bigger molecules though lesser molecular weight dyesdripped away from the peri-arterial space. Different analyses in which solutes, including amyloid- $\beta-40$ were infused directly into brain parenchyma indicated that clearance from the interstitial space happened partly along large central veins [12].

\section{Materials and Methods}

Whit an observational point of view some relevant literature is evaluated in order to produce New strategies to treat some neurodegenerative disease.

All literature in reference is present in PUBMED or other relevant biomedical database.

\section{Results}

All literature founded is reported in reference section.

According the article the Turing machine theory for some spinal cord and brain condition, A toxicological-antidotic depurative approach. J Neurosci Neuron Disord. 2019; 3: 102-134.

Related the bibliography reported in this article a new depurative strategy to treat some spinal cord and brain neuro-degenerative -inflamatory pathology in example ALS SOD mutation and ALZHEIMER D. related can be hypnotized procedure similar to a dyalitic LIKE process or other depurative strategy (in spinal cord place or LCR ). "The wastes of brain metabolism, peroxidation products and glycosylated proteins, accumulate with age-related decreased CSF turnover. Reduction of the CSF turnover rate during ageing leads to accumulation of catabolites in the brain and CSF that are also observed in certain neurodegenerative diseases. Observing the TURING machine theory is possible to verify that a conceptual map make possible to translate from a language to other that seem not related (word War second and ENIGMA machine : secret way of communication). The same is possible to think that an algoritm - machine can traduce some need in response. In example in some neuro-degenerative or inflammatory brain or spinal cord disease a process that can DEPURATE. Form some toxic metabolites or immune-products can delay the progression of some severe disease. Is possible to introduce the hypotesis that a pharmacological, antidotic or medical devices or other physic strategy can help in this setting providing a sustained action during in time or to restore the normal flux of brain wasting system? In an animal model mouse "The results show that there is a reduction in the level of total $A \beta-42$ by $30 \%$ in the group of mice that received the detox gel when compared to the untreated group with a statistical significance $(p<0.001)$ ". Scope of this work is not to produce details of the procedure but that this strategy can be followed. Can a machine better of humans verify all strategy to be used to depurate noble tissue like central nervous system considered so mysterious by the collective meaning? A Computational machine with artificial intelligence can help in choosing the better strategy respecting physiology of this delicate structure, providing the right information about affinity of a new product to the toxic substantia and related to the kinetics of the elimination process."

\section{Discussion and Conclusion}

In this review article, the recent advancements and in vivo experimentations have been summarized which comprise of the glymphatic pathway and meningeal lymphatic vessels as a firmly interlinked waste removal system which proficiently plays its role in the diversified nature of human brain. The glymphatic pathway has rejuvenated the field of CSF transport and featured its function as a component for removal of wastes from the CNS. A huge collection of experimental studies have developed in the course of themost recent years proposing that a adroitly working glymphatic system might be significant for sustainingbrain's wellbeing throughoutthe life. The proposition of slow wave sleep for boosting removal of wastes from brain including amyloidBremoval has accelerated research endeavors aimedat revealing the various components involvedinconserving and regulating the multifaceted system. Imaging studies about envisioning meningeal lymph vessels and CSF transport have addednew data complementingto a starting comprehension of the clearance pathways in the livinganimals and human brain. Furthermore, though meningeal lymphatic vessels are discovered, their absolute limit with respect to waste and fluid clearance is obscure; and they may function as passages for macromolecules and cells having greater molecular weight instead of lessermolecular weight metabolic solute wastes $[20,38,39]$.

Future considerations with increasingly powerful imaging devices be ready to track endogenous waste molecules precisely into brain parenchyma can give additional understanding into the working of these waste removal pathways in human brain in normal health conditions and diseased conditions. 
The evaluative patterns between invertebrates to vertebrates and related vertebrates brain evolution (with new sovra -structure) needed a more complex system to clear the wasting molecule produced.

A better understanding in this phenomena make possible to search new therapeutic strategies

In some neurodegenerative conditions.

\section{Conflict of interests: no}

Clarifications: This work is produced without any diagnostic or therapeutic intent only to produce research hypotesys

\section{References:}

1. Abbott NJ (2013) Blood-brain barrier structure and function and the challenges for CNS drug delivery. Journal of inherited metabolic disease 36: 437-449.

2. Abbott NJ, Patabendige AA, Dolman DE, Yusof SR, Begley DJ (2010) Structure and function of the bloodbrain barrier. Neurobiology of disease 37: 13-25.

3. Achariyar TM, Li B, Peng W, Verghese PB, Shi Y, McConnell E, Benraiss A, Kasper T, Song W, Takano T, Holtzman DM (2016) Glymphatic distribution of CSF-derived apoE into brain is isoform specific and suppressed during sleep deprivation. Molecular neurodegeneration 11: 74 .

4. Brinker T, Stopa E, Morrison J, Klinge P (2014) A new look at cerebrospinal fluid circulation. Fluids and Barriers of the CNS 11: 10.

5. Damkier HH, Brown PD, Praetorius J (2013) Cerebrospinal fluid secretion by the choroid plexus. Physiological reviews, 93: 1847-1892.

6. Davson H, Segal MB (1996) Physiology of the CSF and blood-brain barriers. Boca Raton: CRC 1.

7. Del Zoppo GJ, Moskowitz M, Nedergaard M (2016) The neurovascular unit and responses to ischemia. In Stroke Elsevier 90-101.

8. Di Terlizzi, R Platt S (2006) The function, composition and analysis of cerebrospinal fluid in companion animals: Part I-Function and composition. The Veterinary Journal 172: 422-431.

9. Engelhardt B, Ransohoff RM (2012) Capture, crawl, cross: the T cell code to breach the blood-brain barriers. Trends in immunology 33: 579-589.

10. Hladky SB, Barrand MA (2016) Fluid and ion transfer across the blood-brain and blood-cerebrospinal fluid barriers; a comparative account of mechanisms and roles. Fluids and Barriers of the CNS 13: 19.

11. Iliff JJ, Lee H, Yu M, Feng T, Logan J, Nedergaard M, Benveniste H (2013) Brain-wide pathway for waste clearance captured by contrast-enhanced MRI. The Journal of clinical investigation 123: 1299-1309.

12. Iliff JJ, Wang M, Liao Y, Plogg BA, Peng W, Gundersen GA, Benveniste H, Vates GE, Deane R, Goldman SA, Nagelhus EA (2012) A paravascular pathway facilitates CSF flow through the brain parenchyma and the clearance of interstitial solutes, including amyloid $\beta$. Science translational medicine 4: 147-111.

13. Jessen NA, Munk ASF, Lundgaard I, Nedergaard M (2015) The glymphatic system: a beginner's guide. Neurochemical research 40: 2583-2599.

14. Johnston M, Zakharov A, Papaiconomou C, Salmasi G, Armstrong D (2004) Evidence of connections between cerebrospinal fluid and nasal lymphatic vessels in humans, non-human primates and other mammalian species. Cerebrospinal fluid research 1:2.

15. Koh L, Zakharov A, Johnston M (2005) Integration of the subarachnoid space and lymphatics: is it time to embrace a new concept of cerebrospinal fluid absorption?. Cerebrospinal fluid research 2: 6 .

16. Kress BT, Iliff JJ, Xia M, Wang M, Wei HS, Zeppenfeld D, Xie L, Kang H, Xu Q, Liew JA, Plog BA (2014) Impairment of paravascular clearance pathways in the aging brain. Annals of neurology 76: 845-861.

17. Kulik T, Kusano Y, Aronhime S, Sandler AL, Winn HR (2008) Regulation of cerebral vasculature in normal and ischemic brain. Neuropharmacology 55: 281-288.

18. Mathiisen TM, Lehre KP, Danbolt NC, Ottersen OP (2010) The perivascular astroglial sheath provides a complete covering of the brain microvessels: an electron microscopic 3D reconstruction. Glia 58: 1094-1103.

19. Murtha LA, Yang Q, Parsons MW, Levi CR, Beard DJ, Spratt NJ, McLeod DD (2014) Cerebrospinal fluid is drained primarily via the spinal canal and olfactory route in young and aged spontaneously hypertensive rats. Fluids and Barriers of the CNS 11: 12.

20. Nedergaard M (2013) Garbage truck of the brain. Science 340: 1529-1530.

21. Neuwelt EA, Bauer B, Fahlke C, Fricker G, Iadecola C, Janigro D, Leybaert L, Molnár Z, O'donnell ME, Povlishock JT, Saunders NR (2011) Engaging neuroscience to advance translational research in brain barrier biology. Nature Reviews Neuroscience 12: 169. 
22. Neuwelt EA (2004) Mechanisms of disease: the bloodbrain barrier. Neurosurgery 54: 131-142.

23. Pizzo ME, Thorne RG (2017) The extracellular and perivascular spaces of the brain. In Brain edema. Academic Press 105-127.

24. Prince EA, Ahn SH (2013) September. Basic vascular neuroanatomy of the brain and spine: what the general interventional radiologist needs to know. In Seminars in interventional radiology. Thieme Medical Publishers 30: 234-239.

25. Rennels ML, Gregory TF, Blaumanis OR, Fujimoto K, Grady PA (1985) Evidence for a 'paravascular'fluid circulation in the mammalian central nervous system, provided by the rapid distribution of tracer protein throughout the brain from the subarachnoid space. Brain research 326: 47-63.

26. Rennels ML, Blaumanis OR, Grady PA (1990) Rapid solute transport throughout the brain via paravascular fluid pathways. Advances in neurology 52: 431-439.

27. Syková E, Nicholson C (2008) Diffusion in brain extracellular space. Physiological reviews 88: 12771340.

28. Tait MJ, Saadoun S, Bell BA, Papadopoulos MC (2008) Water movements in the brain: role of aquaporins. Trends in neurosciences 31:37-43.

29. Thorne RG, Nicholson C (2006) In vivo diffusion analysis with quantum dots and dextrans predicts the width of brain extracellular space. Proceedings of the National Academy of Sciences 103: 5567-5572.

30. Thorne RG (2014) Primer on central nervous system structure/function and the vasculature, ventricular system, and fluids of the brain. Drug Delivery to the Brain. Springer: New York 685-706.

31. Thrane AS, Thrane VR, Nedergaard M (2014) Drowning stars: reassessing the role of astrocytes in brain edema. Trends in neurosciences 37: 620-628.

32. Thrane VR, Thrane AS, Plog BA, Thiyagarajan M, Iliff JJ, Deane R, Nagelhus EA, Nedergaard M (2013) Paravascular microcirculation facilitates rapid lipid transport and astrocyte signaling in the brain. Scientific reports $3: 2582$.

33. Trevaskis NL, Kaminskas LM, Porter CJ (2015) from sewer to saviour-targeting the lymphatic system to promote drug exposure and activity. Nature Reviews Drug Discovery 14: 781-803.

34. Xie L, Kang H, Xu Q, Chen MJ, Liao Y, Thiyagarajan
M, O’Donnell J, Christensen DJ, Nicholson C, Iliff JJ, Takano T (2013) Sleep drives metabolite clearance from the adult brain. Science 342: 373-377.

35. Zhang ET, Inman CB, Weller RO (1990) Interrelationships of the pia mater and the perivascular (Virchow-Robin) spaces in the human cerebrum. Journal of anatomy 170: 111.

36. Zhao Z, Nelson AR, Betsholtz C, Zlokovic BV (2015) Establishment and dysfunction of the blood-brain barrier. Cell 163: 1064-1078.

37. Zlokovic BV (2011) Neurovascular pathways to neurodegeneration in Alzheimer's disease and other disorders. Nature Reviews Neuroscience 12: 723.

38. Luisetto M, Ahmadabadi BN, Rafa AY, Sahu RK, Cabianca L (2019) The turing machine theory for some spinal cord and brain condition, A toxicologicalantidotic depurative approach. J Neurosci Neurol Disord 3: 102-134.

39. Mauro, GIbrahim, Oleg Latyschev (2019)TheEvolution of the Nervous System as Model for Search New Pharmacological Strategies in Human Neurological Condition. American Journal of Biomedical Science \& Research 5: 16244-16248 